\title{
Experimental Vehicle Development for Testing Autonomous Vehicle Functions
}

\section{Szilárd Aradi}

Department of Control for

Transportation and Vehicle Systems

Budapest University of

Technology and Economics

Budapest, Hungary

Email: aradi.szilard@mail.bme.hu

\section{Tamás Bécsi}

Department of Control for

Transportation and Vehicle Systems

Budapest Univsersity of

Technology and Economics

Budapest, Hungary

Email: becsi.tamas@mail.bme.hu

\section{Péter Gáspár}

\author{
Systems and Control Laboratory \\ Computer and Automation Research Institute \\ Hungarian Academy of Sciences \\ Budapest, Hungary \\ Email: gaspar.peter@sztaki.mta.hu
}

\begin{abstract}
The paper presents the development of an experimental vehicle framework for testing autonomous vehicle functions. The development of such systems serves two purposes: research and education. On the one hand, such system can be used to aid the development of many driver assistance functionalities. On the other hand it is a good experience for the students on any level of mechatronic education because of its diverse possibilities for mechanical engineering, embedded systems, or control design. Overall system architecture, sensors, actuators and control solutions are outlined in this paper along with the Hardware-in-the-Loop (HIL) testing framework designed for the development.
\end{abstract}

\section{INTRODUCTION AND MOTIVATION}

The automotive industry has always been a leading sector within economy. Innovation is driven by several motives, such as user needs, international regulations, safety requirements and regulations. With the continuous evolution of sensor technologies, information technology, communications and artificial intelligence, new possibilities arise for the vehicle manufacturers. In the early 70 s embedded systems and electronic control began to appear in vehicle electronics and progress in achievable computational performance opened up the possibility to design advanced driver assistance systems (ADAS), such as ABS, ESP, Active and Adaptive Cruise Control, precrash safety systems, etc. Every ADAS solution utilizes the high integration of mechanics, vehicle dynamics and machine intelligence forming a complex mechatronic system. Gietelink et al. [1] classifies the advanced driver assistance systems into five categories by the level of intervention they make:

- Driver information systems, which inform the driver regardless of the current situation e.g. the navigation systems.

- Driver warning systems, which continuously examine and evaluate the current situation but only give warnings, devolving or leaving the responsibility of the decision to the driver. Blind spot detection or Lane departure warning systems are classified in this group. Though such systems do not act instead of the driver, the responsibility of not giving false positive warnings is a requirement since it may distract the driver.
- Intervening systems are a step forward from the warning systems. They take over the control of the vehicle from the driver. Lane keeping, automatic collision avoidance or adaptive cruise control systems fall into this group.

- Integrated passive and active safety systems. Analysing and reacting to the sensor inputs of the vehicle's communication network leads to systems that use active safety data to help prepare passengers before a crash occurs and better mitigate the effects of the collision through seat belt systems and pre-arming airbags. [2]

- Fully automated systems are the Holy Grail of the automotive industry [3], where all driving functions are taken away from the driver. The significance of the field is shown by the fact that not only research centres and all major automotive industry participants have their own autonomous vehicle project but companies from different fields, such as Google have their own research platform. Though autonomous vehicles raise not only technical, but liability and legislative questions [4] to be clear that self-driving cars will spread in the world in the future.

ADAS systems are safety critical applications that must meet the requirements of the ISO standard 26262 (International Standard Road vehicles - Functional safety) [5]. The development process is generally divided into layers of the $\mathrm{V}$ model by the participants of the automotive industry [6]. The development of an ADAS system requires multiple processes concerning functional and safety requirements, system specification, high and module level design, verification, module-integration, system integration and verification and system validation [7]. Each layer is on a different level from mathematical modelling to real application. A development framework includes multiple possibilities of simulation and Hardware-in-the-Loop testing. The developed solutions can be tested on special test vehicles or experimental frameworks.

The paper presents the development of an experimental vehicle framework for testing autonomous vehicle functions. The development of such systems serves two purposes: research and education. 
- Since at the department we focus on educating vehicle engineers who become familiar with mechanical engineering aspects, electronics and embedded systems of the automotive industry it is useful experience for them to participate in the design of a system that requires a wide range of disciplines in their studies.

- Another reason for building a vehicle with sensors and actuators that make it capable of implementing autonomous functions is the varied possibilities it holds for research purposes. Vehicle dynamics and control, route and trajectory planning, sensor fusion, machine vision and intelligence are all research fields the department is interested in.

In the first half of the paper the concept, sensors, actuators and control architecture design are outlined, while the second half presents the Hardware-in-the-Loop development framework of the project.

\section{VEHICLE FRAMEWORK}

\section{A. The vehicle frame}

As mentioned above the purpose of the development is not the building of a real 1:1 scale vehicle, which would raise several questions and needless extra design problems. For the purpose of rapid framework development a much simpler vehicle basis has been chosen: a simple commercial go-cart frame (see Fig. 1.) with hydraulic disc brakes, rigid rear axles and a chain drive.

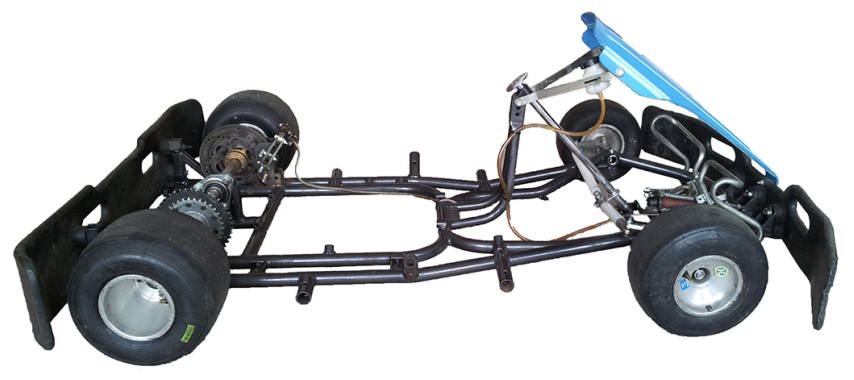

Fig. 1. The go-cart frame used for the project

\section{B. Sensors}

The performance and achievable functions of autonomous vehicles highly depend on their ability to map the environment. The response of the machine intelligence implemented needs precise information about the surroundings of the vehicle. Such systems utilize a wide range of sensors already available in commercial vehicles, such as infra-red or ultrasonic sensors, radars or even vision based solutions. However these elements can not provide all information required. LIDAR systems gained great popularity amongst several self-driving car projects [8][9] because of their ability to provide an accurate depth-of-field map for vehicles. Besides LIDAR, vision-based techniques are common in these vehicles [10][11]. Additional sensors, such as GPS or any other satellite navigation techniques and additional inertial sensors may further enhance performance. Because of the wide range of data sources sensor fusion is a key part in these projects. [12]
As the developed vehicle framework does not use LIDAR, its main advanced sensor is the camera and its vision-based algorithms. It has its own image processing unit, and the required data, such as obstacles, road signs and lane information are provided via their low level description on CAN interface. Besides radar and ultrasonic sensors are mounted on the chassis of the cart.

\section{Actuators}

The system has three main actuators: the driving motor, the steering actuator and the brakes.

An electric motor has been chosen to drive the cart, where three main alternatives seem to be a potential choice:

- Series wound brushed DC motor, with easy controllability, but high wear and maintenance needs;

- Asynchronous motors are widely used in industrial applications, yet the generation of the three-phase power supply needed to drive the motor, control problems and the high investment costs are significant disadvantages of this choice.

- Brushless DC motors have all the advantages of the brushed motors with a slight inconvenience in their control, but the maintenance needs, the reasonable costs and the good power-density ratio makes them the best choice amongst the three.

From the three possibilities the BLDC solution has been chosen: the HPB5000B motor with compact design, water resistant body, stainless steel shaft, and a self-cooling fan [13]. The main technical parameters of the motor are detailed in Table I.

TABLE I. TECHNICAL PARAMETERS OF THE CHOSEN HPM5000B BLDC MOTOR

\begin{tabular}{|c|c|}
\hline Voltage & $48 \mathrm{~V} / 72 \mathrm{~V} / 96 \mathrm{~V} / 120 \mathrm{~V}$ \\
\hline Rated Power & $3 \mathrm{KW}-7.5 \mathrm{KW}$ \\
\hline Efficiency & $91 \%$ \\
\hline Speed & $2000-6000 \mathrm{rpm}$ (customizable) \\
\hline Weight & $11 \mathrm{Kg}$ \\
\hline Height & $126 \mathrm{~mm}$ \\
\hline Diameter & $206 \mathrm{~mm}$ \\
\hline
\end{tabular}

The torque of the motor is transferred to the rear axle by a chain drive with a H428 type chain and a gear ratio of $43 / 18$. Calculations show that the chosen drive is capable of accelerating the vehicle with approximately $2.5 \mathrm{~m} / \mathrm{s}^{2}$ up to $40 \mathrm{~km} / \mathrm{h}$ with the maximum achievable speed of $60 \mathrm{~km} / \mathrm{h}$, which is sufficient for experimental needs.

The installed motor with its controller also has the capability of regenerative braking making the drive able not to accelerate, but decelerate the vehicle. Thus in our design scope the original brake system of the cart is only used as a redundant stopping method for safety and position fixing purposes.

For the purpose of steering two possible motor types can be used, the DC servo and the stepping motor solutions. The minimal torque needed for steering in a cart-type vehicle is approximately $10 \mathrm{Nms}$ while the required angle is $45^{\circ}$. Accurate positioning is a requirement for the steering system and also overload protection is necessary because of the 


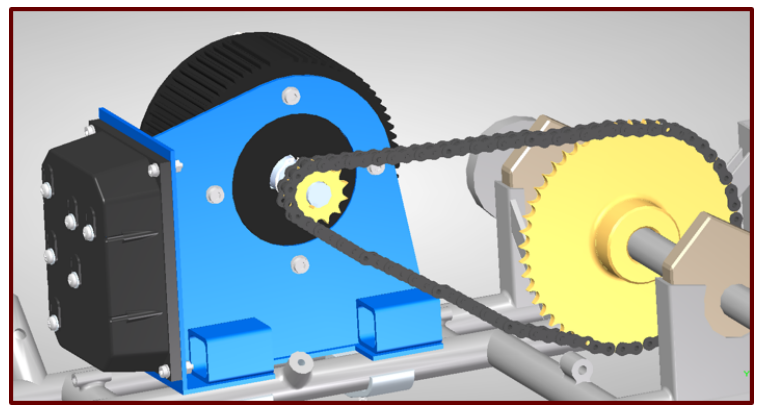

Fig. 2. Drive Chain instalment design

physical circumstances. For this purpose an adjustable torque limiter is used. Despite the higher costs, the stepping motor has been chosen for its ease of instalment and handling, and high precision. SanMotion 103H7823-1740 stepping motors from SANYO-DENKI [14] have been chosen for the steering actuator. This motor has a torque of $2.7 \mathrm{Nm}$ with the rated current of $2 A$. To up-scale torque a gear drive with 4.2 transmission value has been utilized. The design of the steering subsystem is shown in Fig. 3.

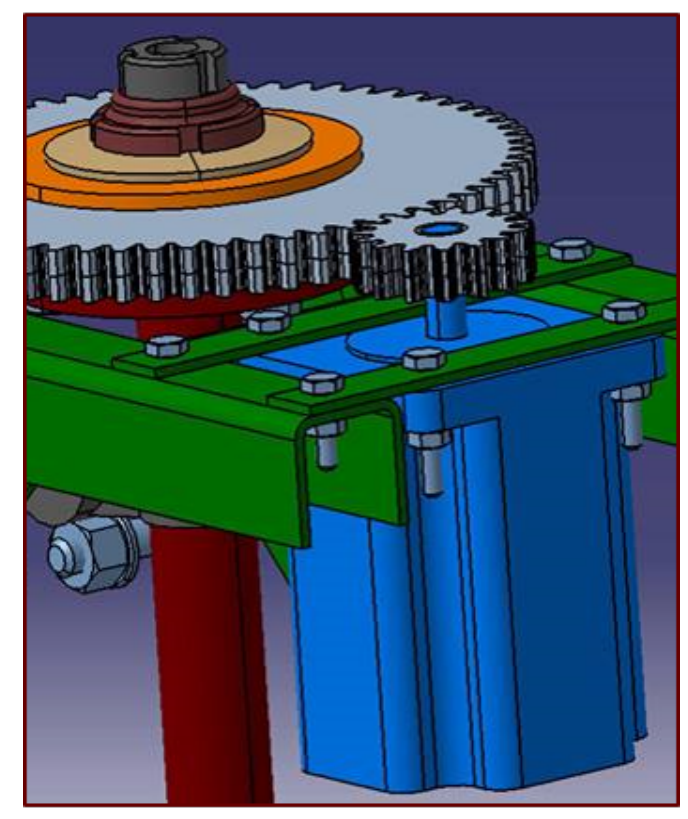

Fig. 3. Steering Actuator instalment design

\section{Control architecture}

The control architecture has been designed to be as modular as possible for the purpose of introducing later changes in the sensor and actuator structure. The separation of control logic and the actuators serves similar aims. Two main Electric Control Units (ECUs) have been designed to operate the vehicle:

- One is for handling sensor information and calculate driving logic,

- the other one for handling the actuators.

The two ECUs are connected with a CAN network where the filtered and processed sensor data are transferred along with the control commands. The illustration of the logical specification of the control architecture is shown in Fig. 4.

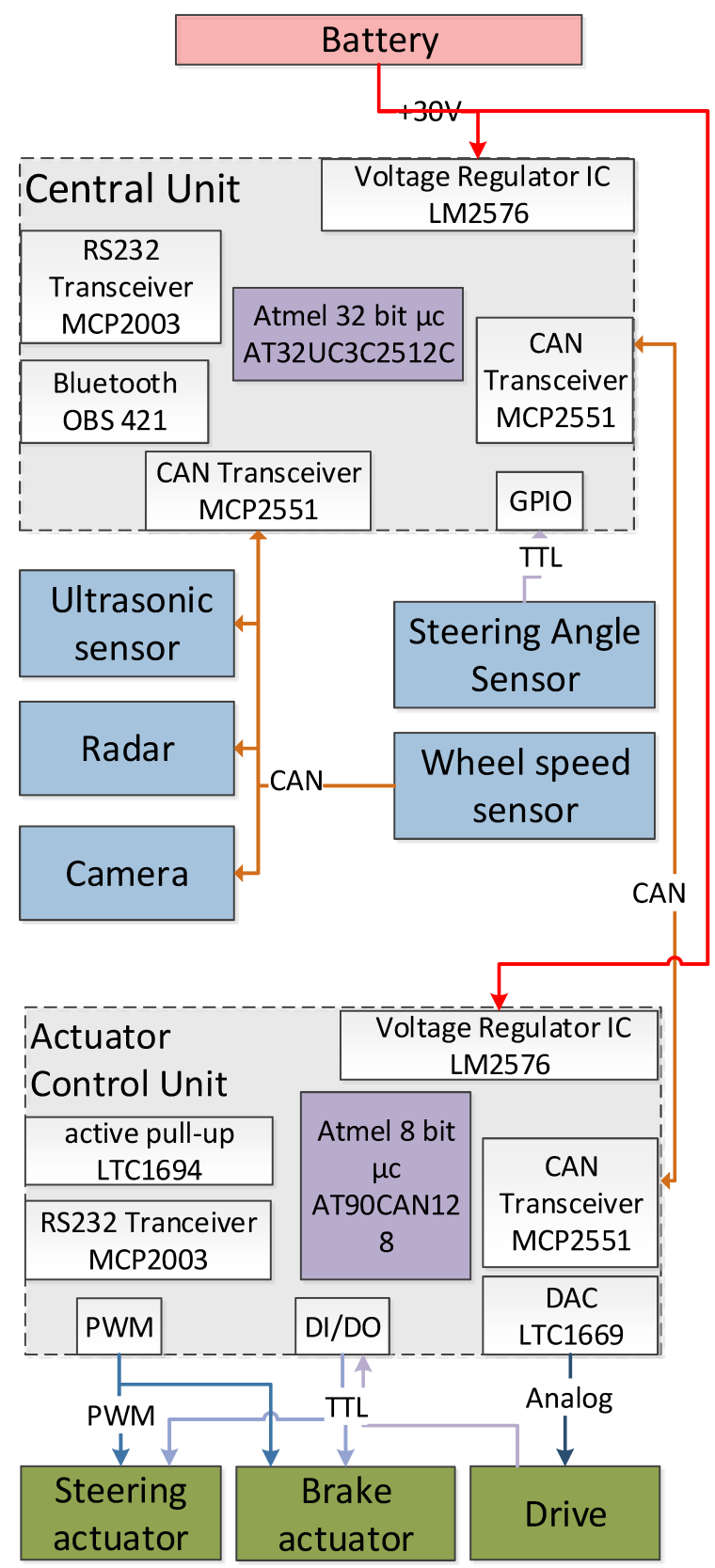

Fig. 4. Control architecture logical specification

The actuator control unit handles the drive modules of the steering, brake, and drive actuators generating the pulse-width modulated and analogue control signals for them to follow the reference provided by the central unit. This ECU manages simple control loops and needs relatively low computational performance. The core of the unit is an Atmel AT90CAN128 [15] micro-controller, which is a low-power 8-bit AVR RISCbased unit with all required analogue and digital I/Os and a V2.0A/V2.0B compliant CAN controller. 
The central control unit has more complex tasks, since it must handle two main tasks. First the central unit collects all sensor information. Direct connection to all sensors may have been obvious but that way modularity needs would have been compromised. To resolve this problem all sensors provide data via a CAN network and those that do not have a CAN interface receive a simple converter to connect to this network. The other function of the central ECU is to calculate the driving tasks mainly based on camera information.

The already implemented functions are:

- Lane following at a fix speed,

- Object avoidance.

Because of the high computational needs of the central unit, an AT32UC3C2512C microcontroller has been chosen. The AT32UC3C family is a complete System-On-Chip controller based on the AVR32UC RISC processor running at frequencies up to $66 \mathrm{MHz}$. It is designed for cost-sensitive embedded applications, with particular emphasis on low power consumption, high code density and high performance. [16]

\section{HARDWARE IN THE LOOP TESTING}

The development process of even a relatively small scale autonomous vehicle poses several feasibility problems regarding the iterative process of function design and testing. The project has numerous subtasks whose progress can not be handled in a linear way, thus parallel development is needed to achieve advance. Yet the developed function must be tested, first at model level and then at the implementation level with the final embedded system.

\section{A. Theoretical control loop}

The simplest theoretical control loop of the system has four main elements:

- The vehicle, including its dynamics with regard to the actuators and the wheel-road interaction.

- The environment possessing the road, signs, other vehicles and objects. From a modelling point of view the necessary visualization part of model building is also included.

- The sensor system, with special regard to vision-based detection since the camera itself has an important role and a significant amount of development requirements.

- $\quad$ Last there is the controller with the implemented logic.

Naturally individual development and testing can be performed an all subsystems by using previously defined raw data or simple emulation. The problem arises at the level of subsystem synthesis. On the one hand the different development progress of the subtasks prevents overall testing, on the other hand for full system evaluation a track is needed.

These together lead to the application of a modular Hardware-in-the-Loop (HIL) [17][18] framework, where all system parts can be easily exchanged by any of its three representation:

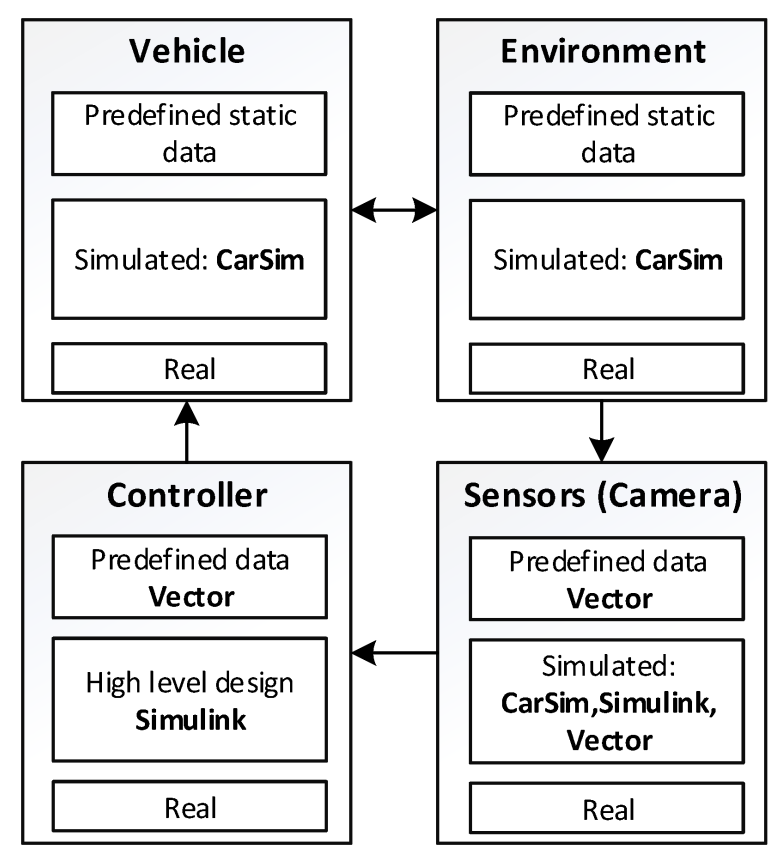

Fig. 5. The modular possibilities of Harware-in-the-Loop development

- Previously defined data or in some cases simple computer model.

- Simulated behaviour with detailed mathematical representation.

- Real system/reality

Naturally, not all combinations of these three possibilities at all four subsystem form useful test cases. Fig. 5 shows the stylized control loop with the possible simulation replacements.

\section{B. Simulation elements of the HIL process}

The simulation elements of the Hardware-in-the-Loop testing and development are shown in Fig. 6.

For the simulation of vehicle dynamics, vehicle-road interaction and 3D visualization as simulated input for the camera the CarSim software family has been used. CarSim simulates the dynamic behaviour of passenger cars, race cars, light trucks, and utility vehicles. It animates simulated tests and its outputs are the calculated variables in real-time [19].

For the design of high-level control systems Matlab/Simulink has been used because of its ease of use and high interfacing capabilities to CarSim.

CAN communication is managed by the CANCase XL, a professional CAN/LIN/J1708 bus interface with USB 2.0 interface from Vector $\mathrm{GmbH}$.

Fig. 7 shows an example set-up, where the vehicle model and $3 \mathrm{~d}$ visualization are simulated with CarSim, whilst the 


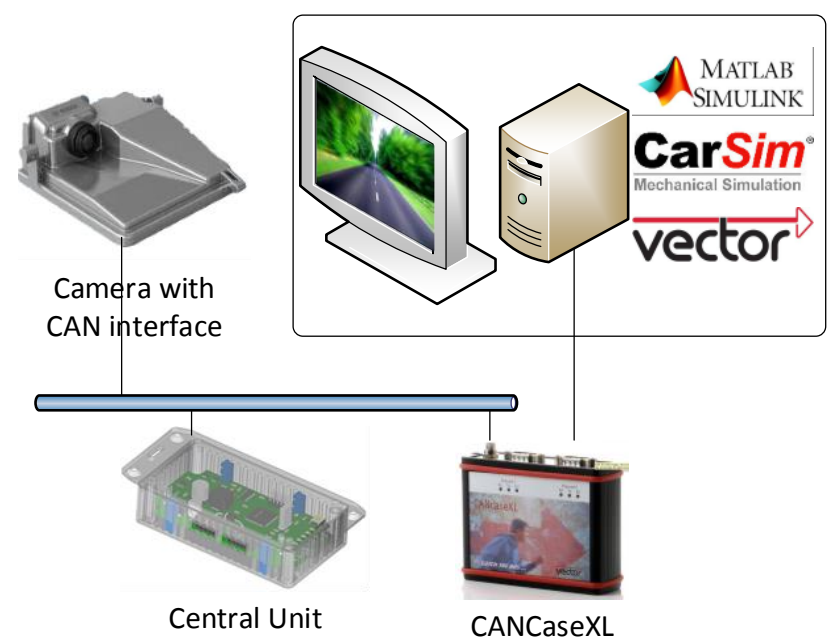

Fig. 6. Simulation elements of the HIL process

real camera understands the projected image, transfers data to the Control ECU, whose control signals are looped back to CarSim with a simple Simulink interface.

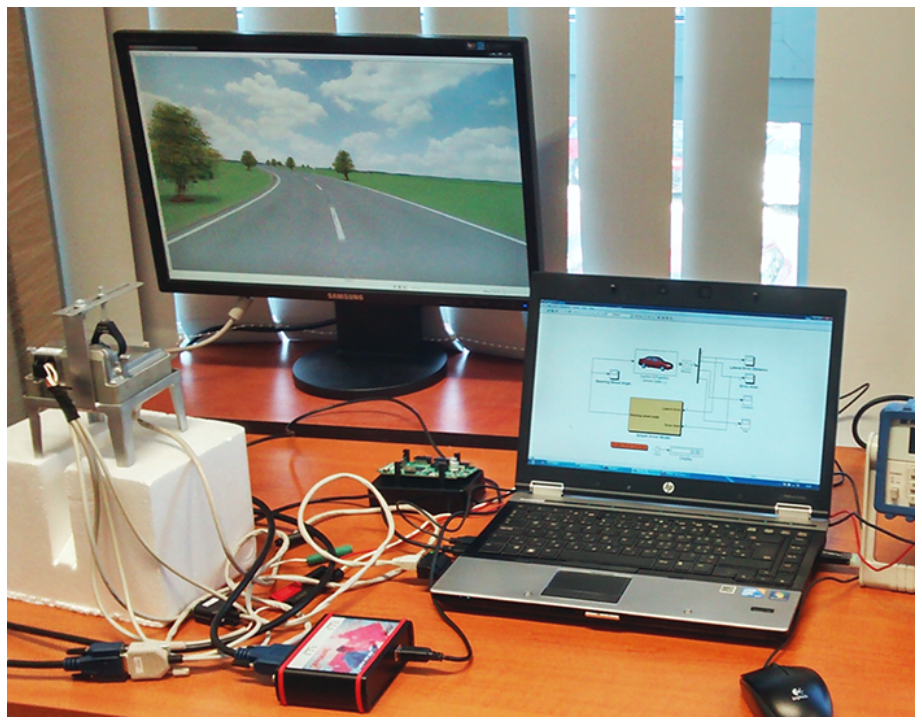

Fig. 7. Example Hardware-in-the-Loop configuration

\section{CONCLUSIONS}

The paper has presented an experimental vehicle framework based on a laboratory vehicle. The design of the system aims to develop a modular structure in which sensors and actuators can be easily interchanged when the scope of the high level function development changes. Several driver assistance solutions can be developed and tested with the usage of this relatively small scale vehicle, while our future plans are aimed at the development of autonomous functions. These envisioned goals can be achieved on different levels with the framework. Engineering students can work on comparatively lower complexity level problems, such as lane detection and keeping, while in the research intelligent vehicle solutions and self driving capabilities are targeted. A vehicle framework by itself is not sufficient for the overall development process therefore a Hardware-in-the-Loop system is also introduced to aid the entire process on all design levels.

\section{ACKNOWLEDGMENT}

The research has been conducted as part of the project TÁMOP-4.2.2.A-11/1/KONV-2012-0012: Basic research for the development of hybrid and electric vehicles. The Project is supported by the Hungarian Government and co-financed by the European Social Fund.

\section{REFERENCES}

[1] O. Gietelink, J. Ploeg, B. De Schutter, and M. Verhaegen, "Development of advanced driver assistance systems with vehicle hardware-in-the-loop simulations," Vehicle System Dynamics, vol. 44, no. 7, pp. 569-590, 2006.

[2] S. Tokoro, K. Moriizumi, T. Kawasaki, T. Nagao, K. Abe, and K. Fujita, "Sensor fusion system for pre-crash safety system," in Intelligent Vehicles Symposium, 2004 IEEE, June 2004, pp. 945-950.

[3] P. Marks, "Autonomous cars ready to hit our roads," New Scientist, vol. 213, no. 2858, pp. 19-20, 2012.

[4] J. K. Gurney, "Sue my car, not me: Products liability and accidents involving autonomous vehicles," Journal of Law, Technology and Policy, vol. 2013, no. 2, p. 101, 2013.

[5] ISO, "International standard road vehicles - functional safety," International Organization for Standardization, Geneva, Switzerland, ISO 26262, 2011.

[6] R. Rana, M. Staron, C. Berger, J. Hansson, M. Nilsson, and F. Törner, "Increasing efficiency of iso 26262 verification and validation by combining fault injection and mutation testing with model based development," in 8th International Joint Conference on Software TechnologiesICSOFT-EA, Reykjavík, Iceland, July 2013, 2013, pp. 251-257.

[7] M. Broy, "Challenges in automotive software engineering," in Proceedings of the 28th international conference on Software engineering. ACM, 2006, pp. 33-42.

[8] J. Levinson, J. Askeland, J. Becker, J. Dolson, D. Held, S. Kammel, J. Z. Kolter, D. Langer, O. Pink, V. Pratt et al., "Towards fully autonomous driving: Systems and algorithms," in Intelligent Vehicles Symposium (IV), 2011 IEEE. IEEE, 2011, pp. 163-168.

[9] T. Luettel, M. Himmelsbach, and H.-J. Wuensche, "Autonomous ground vehicles, concepts and a path to the future," Proceedings of the IEEE, vol. 100, no. Special Centennial Issue, pp. 1831-1839, 2012.

[10] C. J. Taylor, J. Košecká, R. Blasi, and J. Malik, "A comparative study of vision-based lateral control strategies for autonomous highway driving," The International Journal of Robotics Research, vol. 18, no. 5, pp. 442453, 1999.

[11] M. Bertozzi, A. Broggi, and A. Fascioli, "Vision-based intelligent vehicles: State of the art and perspectives," Robotics and Autonomous Systems, vol. 32, no. 1, pp. 1-16, 2000.

[12] D. Gohring, M. Wang, M. Schnurmacher, and T. Ganjineh, "Radar/lidar sensor fusion for car-following on highways," in Automation, Robotics and Applications (ICARA), 2011 5th International Conference on. IEEE, 2011, pp. 407-412.

[13] High power bldc motors, hpm5000b. Golden Motor Technology Co Ltd. [Online]. Available: http://www.goldenmotor.com/hubmotors/

[14] Stepping Motors data sheet, SanMotion 103H7823-1740, SANYO DENKI CO.,LTD.

[15] AT90CAN32/64/128 Complete Datasheet, Atmel Corporation, 2008.

[16] Atmel AVR 32-bit Architecture Manual Complete, Atmel Corporation, 2012.

[17] W. Deng, Y. Lee, and A. Zhao, "Hardware-in-the-loop simulation for autonomous driving," in Industrial Electronics, 2008. IECON 2008. 34th Annual Conference of IEEE, Nov 2008, pp. 1742-1747.

[18] D. J. Verburg, A. C. Van der Knaap, and J. Ploeg, "Vehil: developing and testing intelligent vehicles," in Intelligent Vehicle Symposium, 2002. IEEE, vol. 2. IEEE, 2002, pp. 537-544.

[19] CarSim, Mechanical Simulation Corporation, 2013. 